\title{
Treatment of Leprosy with B 663 Appraisal of the Pilot Trial after three years
}

\author{
S. G. BROWNE, M.D., F.R.C.P., F.R.C.S., D.T.M. \\ Leprosy Service Research Unit, Uzuakoli, Eastern Nigeria
}

The preliminary and supplementary reports of the pilot trial of B 663 conducted at Uzuakoli (Browne and Hogerzeil i $962 \mathrm{a}, \mathrm{b}$ and c) offered evidence that the drug is active in human leprosy and that its action is probably enhanced by the addition of dapsone.

The present report summarizes the subsequent progress of the same group of patients three years after they had begun six or I 2 months' treatment with B 663. (Some of them have now been under observation for four years.)

\section{The patients}

The patients, unselected and untreated, were placed on treatment in the order of admission. Of the total of 28,26 had lepromatous leprosy and the remaining two had borderline leprosy highly positive bacteriologically. One patient died during the $13^{\text {th }}$ month from a disease unconnected with leprosy.

\section{Treatment}

I. Twenty-two patients had six months' treatment with B 663; during this period:

Seven had no other drug;

ten had standard doses of dapsone;

five had standard doses of dapsone, together with ditophal (Etisul, I.C.I.) initially for three months.

2. Five patients had I 2 months' treatment with B 663 alone.

At the end of the period of treatment with B 663, patients in all groups received standard doses of dapsone.

\section{Dose/body-rveight}

Twenty patients whose body-weight averaged I 8 lb. (53.6 kg.) received $300 \mathrm{mg}$. B 663 daily, or $5.6 \mathrm{mg}$. per $\mathrm{kg}$. body-weight.

Seven patients whose body-weight averaged $87 \frac{1}{2} \mathrm{lb}$. (39.7 kg.) received $200 \mathrm{mg}$. B $66_{3}$ daily, or $5.0 \mathrm{mg}$. per $\mathrm{kg}$. body-weight.

One patient weighing $50 \mathrm{lb}$. (22.7 kg.) received I oo mg. B 663 daily, or $4.4 \mathrm{mg}$. per $\mathrm{kg}$. body/weight.

The weight of drug given daily per kg. ranged from $4.4 \mathrm{mg}$. to $6.8 \mathrm{mg}$.

Results

To supplement earlier published reports, the following assessment was made at the end of the $3^{6 \text { th }}$ month, counting from the beginning of treatment with B 663 .

\section{Clinical}

All patients in all groups showed marked objective improvement, but no significant differences could be detected between the groups. From examination priricipally of the skin lesions (nodules, macules, diffuse infiltration) and peripheral nerves, the improvement was assessed as follows: excellent 20, very good 6, good I .

\section{Skin lesions}

The degree of permanent macroscopic damage to the skin was proportional to the extent and more especially to the depth of the initial involvement. Young nodules and recently developed macular areas healed without visible trace, but lesions of long standing healed with obvious fibrosis and atrophy of the elastic subcutaneous tissue.

\section{Nerve lesions}

The slight nerve involvement seen early in lepromatous leprosy proved to be clinically reversible, but contractures and paralyses due to permanent nerve damage were unaffected by treatment with B 663. Some of the patients did not present themselves for treatment until probably three to five years after the clinically recognizable onset of the disease, when clawing, 
peripheral sensory impairment and neuropathic ulceration had already made their appearance; in point of fact, it was frequently these complications that brought the patients to the Diagnostic Clinic. It is reasonable to infer that the rapidity of action of $\mathrm{B} 663$, alone or in conjunction with dapsone, coupled with the absence of acute exacerbation (including erythema nodosum leprosum and polyneuritic episodes) during treatment with $\mathrm{B}$ 663, prevented the nerve damage that would be expected to occur in such a group under other standard therapeutic regimes. Similarly, the development of iritis and iridocyclitis was probably forestalled.

\section{Skin pigmentation}

In the course of treatment, the skin became initially red and subsequently blackish. These changes were symptomless, and apparently without pathological significance. The red colour disappeared generally within six months of cessation of treatment with $\mathrm{B} 66_{3}$, but the hyperpigmentation persisted for rather longer. Both the red and the black coloration might prove unacceptable in the lighter-hued, but the dark-skinned patients in this trial raised no objections.

\section{Bacteriological}

Normal-staining solid rods of $M$. leprae disappeared from the routine skin smears taken regularly from eight sites in skin and nasal mucosa between the 28th and the 52 nd weeks, the disappearance being most rapid in the group taking B 663 and dapsone. The initial average percentage of normal-staining bacilli $(42-52)$ was approximately the same for all groups.

An analysis based on the dosage of B 663/ body-weight ratio showed no appreciable difference in the time necessary for complete disappearance of normal-staining forms between the high, medium and low dose/body-weight ratios, i.e. the speed did not seem to vary with the concentration of B 663. Analysed in this fashion, the average time taken lay between 32 and $3^{8}$ weeks. In I 5 patients with a high (i.e. over 5I) initial Morphological Index (i.e. percentage of normally staining forms), the Index fell to zero in an average of 36 weeks, whereas in the remaining i 2 patients, whose average Index was 24 , the Index fell to zero in 32 weeks.

\section{Reappearance of morphologically normal forms}

of M. leprae

The more or less sudden reappearance of a varying proportion of solid-staining rods (of $M$. leprae) at some or all the eight sites routinely smeared, accompanied generally by a rise in the Bacterial Index, after I 2 months' treatment with B 663 alone (Browne and Hogerzeil i 962 c) was a transient phenomenon, the excess mycobacterial load (accounted for by the solidstaining rods) gradually disappearing in the course of three or four months. The eventual bacteriological and clinical improvement in these patients seemed unaffected by this episode.

\section{Bacterial clearance}

It is evident that the speed of removal of effete bacilli depends on factors unconnected with the bactericidal or bacteriostatic action of a drug that is apparently active in human leprosy. Apart from three patients suffering from exacerbation (developing during treatment with dapsone), no patient at the 36 th month had a Bacterial Index of 0.5 or over (maximum: 4.0); in the 12 patients with a positive Index, the bacilli were very degenerate. In the remaining I5, no acid-fast material could be seen in any of the eight smears taken at monthly intervals by a standard technique.

The speed of clearance in the patients of the group that had been given dapsone in addition to B 663 was slightly greater than that of the others. No differences were noted between the patients on high, medium or low B 663/bodyweight ratios.

The consistent and continuous decrease in the Bacterial Index in all groups was a gratifying feature of the treatment given.

\section{Possible anti-inflammatory action of $B 663$}

It was observed that while taking $B \quad 66_{3}$, the patients appeared much less liable to experience episodes of acute exacerbation, though subsequent experience when they were receiving standard doses of dapsone indicates that as a group they were typically liable to such episodes. It seems possible that in these circumstances B 663 exerts a positive suppressive or antiinflammatory action. 


\section{H IS TOLOGY}

The improvement under therapy was consistent and rapid.

Sections taken before treatment showed lepromatous leprosy of a degree consistent with the clinical findings.

Within eight months, the numbers of foamy cells decreased considerably, to be replaced by a granulomatous infiltrate composed mainly of histiocytes. A noteworthy feature in several sections was the scanty character of this infiltration. Pari passu, the amount of collagen in the dermis increased.

The nerves were, typically, uninvolved in the cellular response; in many of them $M$. leprae could be seen, either singly or in groups lying between the nerve fibres.

Bacilli soon began to show gross morphological changes. Within six to eight months, all were fragmented, including those within the dermal nerve fibrils, and a high proportion had disappeared.

The conclusion reached on the histological appearances was that the response to treatment was very satisfactory. The typical lepromatous picture was rapidly modified, coming to resemble that of indeterminate leprosy.

\section{Patients' reactions}

Without exception, the patients taking B 663 are enthusiastic about the drug. Many other patients, having noted the progress of their fellows in this trial, have asked to be given the drug.

\section{CONCLUSIONS}

This group of patient with severe bacilliferous leprosy improved more consistently and more rapidly than any similar group in the writer's experience.

In the doses given, B 663 has an undoubted action in lepromatous and borderline leprosy, leading to relatively rapid clinical and bacteriological improvement.

While taking the drug, patients seem to be much less liable to episodes of acute exacerbation.
Consistent with the rapid improvement, the risk of nerve and eye damage appears to be reduced.

The red coloration of the skin and the subsequent hyperpigmentation are transitory and symptomless phenomena of no pathological significance, and did not constitute a contraindication among the more deeply pigmented patients participating in this trial.

Since improvement was equally noticeable in patients having relatively low doses in relation to body-weight, it may be that doses smaller than those used in this trial may suffice to ensure clinical improvement and bacteriological progress, and at the same time still obviate acute exacerbation.

Attempts should be made to prevent the emergence of resistant forms by giving another drug in addition to B $66_{3}$, such as dapsone, or isoniazid, as advocated by Chang (I 962). The additive effect may accelerate not only the rate of disappearance of normal-staining forms but also the clearance of non-viable and fragmented bacilli.

\section{A GKNOWLEDGEMENTS}

My thanks are due to Messrs J. R. Geigy, s.A., of Basle, for generous supplies of their product B 663 and much helpful advice; to Messrs I.C.I. (Pharmaceuticals) Ltd, for supplies of 'Etisul' (ditophal); to Dr R. G. Cochrane, of the Leprosy Research Unit, London, for muchappreciated help in the histological evaluation of the changes observed in biopsy specimens; and to Dr S. O. Egwuatu, Chief Medical Officer, Ministry of Health, Eastern Nigeria, for permission to publish this article.

\section{REFERENCES}

BRowne, s. G. and hogerzeil, L. M. (I962). Leprosy Rev., 33, 6 .

Browne, s. G. and hogerzeil, L. M. (i 962). Leprosy Rev., 33, I 82 .

Browne, s. G. and hogerzeil, L. M. (1962). Leprosy Rev., 33, I 85 .

Chang, u. T. (1962). Antimicrobial Agents and Chemotherapy ( 1962$), 294$. 